\title{
Prompt signals and displaced vertices in sparticle searches for next-to-minimal gauge-mediated supersymmetric models
}

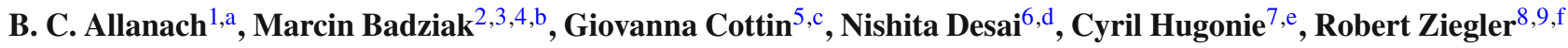 \\ ${ }^{1}$ Department of Applied Mathematics and Theoretical Physics, Centre for Mathematical Sciences, University of Cambridge, Wilberforce Road, \\ Cambridge CB3 0WA, UK \\ 2 Institute of Theoretical Physics, Faculty of Physics, University of Warsaw, ul. Pasteura 5, 02-093 Warsaw, Poland \\ ${ }^{3}$ Department of Physics, University of California, Berkeley, CA 94720, USA \\ ${ }^{4}$ Ernest Orlando Lawrence Berkeley National Laboratory, University of California, Berkeley, CA 94720, USA \\ ${ }^{5}$ Cavendish Laboratory, University of Cambridge, 19 JJ Thomson Ave, Cambridge CB3 0HW, UK \\ ${ }^{6}$ Institut für Theoretische Physik, Philosophenweg 16, 69120 Heidelberg, Germany \\ ${ }^{7}$ LUPM, UMR 5299, CNRS, Université de Montpellier, 34095 Montpellier, France \\ ${ }^{8}$ Sorbonne Universités, UPMC Univ Paris 06, UMR 7589, LPTHE, 75005 Paris, France \\ ${ }^{9}$ CNRS, UMR 7589, LPTHE, 75005 Paris, France
}

Received: 27 June 2016 / Accepted: 19 August 2016 / Published online: 1 September 2016

(c) The Author(s) 2016. This article is published with open access at Springerlink.com

\begin{abstract}
We study the LHC phenomenology of the nextto-minimal model of gauge-mediated supersymmetry breaking, both for Run I and Run II. The Higgs phenomenology of the model is consistent with observations: a $125 \mathrm{GeV}$ standard model-like Higgs which mixes with singlet-like state of mass around $90 \mathrm{GeV}$ that provides a $2 \sigma$ excess at LEP II. The model possesses regions of parameter space where a longer-lived lightest neutralino decays in the detector into a gravitino and a $b$-jet pair or a tau pair resulting in potential displaced vertex signatures. We investigate current bounds on sparticle masses and the discovery potential of the model, both via conventional searches and via searches for displaced vertices. The searches based on promptly decaying sparticles currently give a lower limit on the gluino mass $1080 \mathrm{GeV}$ and could be sensitive up to $1900 \mathrm{GeV}$ with $100 \mathrm{fb}^{-1}$, whereas the current displaced vertex searches cannot probe this model due to $b$-quarks in the final state. We show how the displaced vertex cuts might be relaxed in order to improve signal efficiency, while simultaneously applied prompt cuts reduce background, resulting in a much better sensitivity than either strategy alone and motivating a fully fledged experimental study.
\end{abstract}

\footnotetext{
a e-mail: b.c.allanach@damtp.cam.ac.uk

be-mail: mbadziak@fuw.edu.pl

c e-mail: gfc24@cam.ac.uk

de-mail: n.desai@thphys.uni-heidelberg.de

e e-mail: cyril.hugonie@umontpellier.fr

f e-mail: robert.ziegler@1pthe.jussieu.fr
}

\section{Introduction}

Sparticle searches at the large hadron collider (LHC) have so far yielded no clear discovery. Strengthening exclusion limits [1] on the masses of sparticles in the minimal supersymmetric standard model (MSSM) mean that points in the model parameter space with low fine-tuning have been ruled out. In particular, the ATLAS and CMS experiments have measured a particle whose properties are compatible with a standard model (SM) Higgs of mass around $125 \mathrm{GeV}$. While such a mass is still compatible with the theoretical upper bound in the MSSM, it is rather on the heavy side and corresponds to a fairly heavy stop mass, which in turn induces lower bounds on typical quantifications of fine-tuning in the electroweak symmetry breaking sector (see e.g. Ref. [2]). Arguably, it may be important to consider non-minimal supersymmetric scenarios that can alter the interpretation of standard sparticle searches, perhaps allowing regions with lower finetuning than in minimal scenarios. However, one may get an impression from "simplified model searches" (where the MSSM spectrum is set to be heavy except for a few sparticles relevant for a particular search) that strongly interacting particles with multi- $\mathrm{TeV}$ masses are already ruled out (see, for example Ref. [1]), eliminating the low fine-tuning regions. Still, in more realistic non-simplified MSSM scenarios, points in parameter space exist with gluino masses of $700 \mathrm{GeV}$ or squark masses of $500 \mathrm{GeV}$ which evade all Run I sparticle searches [3] or even which evade all 2015 Run II searches [4] with consequently fairly low values of fine-tuning. 
Non-simplified MSSM scenarios are suggested by well motivated ultra-violet scenarios of SUSY breaking, for example Gauge Mediation. While gauge-mediated SUSY breaking provides a neat solution to the SUSY flavour problem (i.e. the absence of large sources of flavour violation in the soft terms), its minimal realisations are in trouble because they typically predict a SM-like Higgs mass that is too low compared to the observed value around $125 \mathrm{GeV}$. A potentially fruitful path was explored by introducing additional dynamics to increase the SM-like Higgs boson mass prediction while maintaining fairly low levels of fine-tuning, see Refs. [5-25].

In Ref. [26], we revisited a simple model by Delgado et al. [27] (DGS) that combines gauge mediation (GM) and the next-to-minimal supersymmetric standard model (NMSSM). The field content of the model is the one of the NMSSM, plus two copies of messengers in $\mathbf{5}+\overline{\mathbf{5}}$ representations of SU(5), denoted by $\Phi_{i}, \bar{\Phi}_{i}$, respectively ( $i \in$ $\{1,2\})$, with doublet and triplet components $\Phi_{i}^{D}, \bar{\Phi}_{i}^{D}$ and $\Phi_{i}^{T}, \bar{\Phi}_{i}^{T}$. SUSY breaking is parameterised by the spurion $X=M+F \theta^{2}$ (where $M$ is the messenger scale and $\theta$ is the Grassmann valued $N=1$ superspace coordinate). Aside from Yukawa interactions, the superpotential contains spurion-messenger couplings and singlet $S$-messenger couplings (first introduced in the context of gauge mediation in Ref. [28]):

$$
\begin{aligned}
W= & \cdots+\lambda S H_{u} H_{d}+\frac{\kappa}{3} S^{3} \\
& +X \sum_{i}\left(\kappa_{i}^{D} \bar{\Phi}_{i}^{D} \Phi_{i}^{D}+\kappa_{i}^{T} \bar{\Phi}_{i}^{T} \bar{\Phi}_{i}^{T}\right) \\
& +S\left(\xi_{D} \bar{\Phi}_{1}^{D} \Phi_{2}^{D}+\xi_{T} \bar{\Phi}_{1}^{T} \Phi_{2}^{T}\right),
\end{aligned}
$$

where the singlet-messenger couplings unify at the grand unified theory scale $M_{\mathrm{GUT}}: \xi_{D}\left(M_{\mathrm{GUT}}\right)=\xi_{T}\left(M_{\mathrm{GUT}}\right) \equiv \xi$ with unified coupling $\xi$. The scale of the SUSY breaking terms is fixed by the parameter $\tilde{m}=1 /\left(16 \pi^{2}\right) F / M$.

It was shown in Ref. [26] that in the DGS model one can obtain a $125 \mathrm{GeV}$ standard model-like Higgs boson with stops as light as $1.1 \mathrm{TeV}$, thanks to the mixing of the Higgs with a singlet state at $\mathcal{O}(90-100) \mathrm{GeV}$ which is compatible with LEP data [29]. With these Higgs constraints, essentially all parameters are fixed except for the GM messenger scale which mainly controls the phenomenology of the gravitino. The central feature of the model, apart from the light Higgs that might explain the LEP excess [30] is the peculiar structure of the light sparticle spectrum. The lightest sparticle (LSP) is the gravitino ${ }^{1} \tilde{G}$ with mass and couplings effectively set by the GM messenger scale, the

\footnotetext{
1 Another attractive feature of the NMSSM realisation of gauge mediation is that the singlet allows the gravitino to be a good dark matter candidate even for large reheating temperatures that are compatible with thermal leptogenesis [31].
}

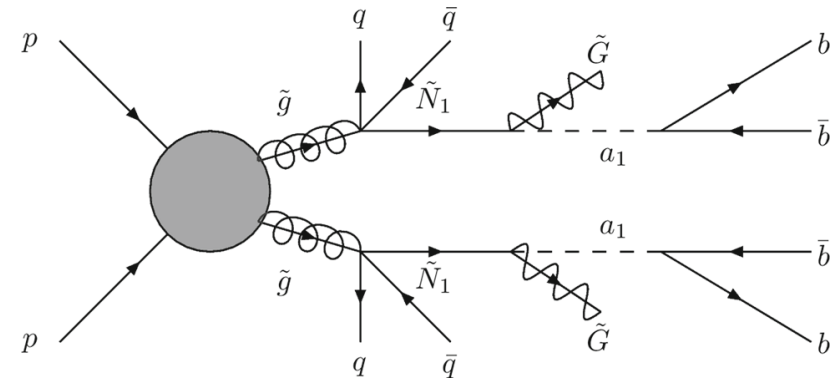

Fig. 1 An example of LHC sparticle production in the DGS model, followed by sparticle decay. In this example, we have four hard prompt jets from gluinos decaying into quarks $q$ and anti-quarks $\bar{q}$; the lightest neutralino $\tilde{N}_{1}$ may have an intermediate lifetime, producing displaced vertices, each generating $b \bar{b}$. The gravitino $\tilde{G}$ leaves a missing transverse momentum signature. The lightest pseudo-scalar $a_{1}$ has a lower branching ratio for decays into $\tau \bar{\tau}$ than $b \bar{b}$. The $\tilde{g} \rightarrow \tilde{N}_{1}$ part of the decay may commonly be more complicated, involving a cascade decay and concomitant additional SM states

next-to-LSP (NLSP) is a singlino-like neutralino $\tilde{N}_{1}$ of mass around $100 \mathrm{GeV}$, and the next-to-NLSP (NNLSP) is a binolike neutralino $\tilde{N}_{2}$ or stau $\tilde{\tau}$, depending on the GM messenger scale. The presence of the singlino alters SUSY decay chains as compared to the MSSM, leading to additional $b$ jets or taus. One distinctive feature of this scenario is that the singlino decays to a gravitino and a light singlet-like pseudo-scalar $a_{1}$ of mass around $20 \mathrm{GeV}$, with the latter decaying predominantly to $b \bar{b}$ as well as to $\tau \tau$. Depending on the GM messenger scale, the two $b$-jets may be produced far outside the detector (when the $\tilde{N}_{1}$ is quasi-stable, at high GM scales) or at low GM scales, they may be produced within the detector from displaced vertices (DVs). This peculiar feature of a long-lived singlino decay was already noticed in Ref. [27]. An example diagram showing LHC sparticle production in the model is shown in Fig. 1.

In this paper, we wish to evaluate the collider phenomenology of the model. In Sect. 2, we describe our benchmark model, and describe the tools used for simulation of the signal events and validation of our analysis. In Sect. 3, we recast the most constraining prompt sparticle searches from the LHC in order to find how stringent the bounds on the model are and then we estimate the future reach. In Sect. 4, we detail a study of DV signatures, starting with recasting the current ATLAS multi-track DV + jets analysis and showing that current searches are not sensitive to our model. By changing the cuts, we suggest ways in which the DV cuts can be loosened, and how cuts on accompanying hard prompt objects can be used to combat background rates. In Sect. 4.4, we estimate the search reach from early Run II data with such a strategy. After a summary and discussion in Sect. 5, we define the relevant DV variables $d_{0}$ and $r_{\mathrm{DV}}$ in Appendix A, for easy reference by the reader. 


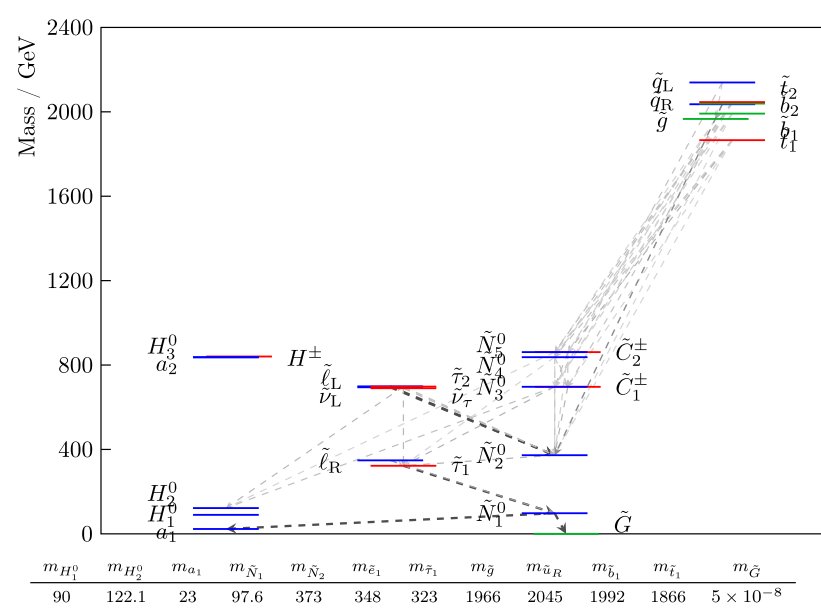

Fig. 2 Spectrum and the more likely sparticle decays of benchmark point P0: $\xi=0.01, \lambda\left(M_{\text {SUSY }}\right)=0.009, M=1.4 \times 10^{6} \mathrm{GeV}$, $\tilde{m}=863 \mathrm{GeV}$ and $\tan \beta=28.8$. Decays into sparticles which have a branching ratio greater than $10 \%$ are displayed by the arrows. The figure was produced with the help of PySLHA3 . 0.4 [34]. The table shows some more precise details of the spectrum of the benchmark point $\mathrm{P} 0$. All masses are listed in $\mathrm{GeV}$ units and the lightest neutralino has a decay length $c \tau_{\tilde{N}_{1}}=99 \mathrm{~mm}$

\section{Benchmark model and event generation}

Here, we discuss the limits from the LHC, both in prompt and displaced searches. Like minimal gauge mediation in the MSSM, the DGS model also needs large radiative SUSY corrections to obtain the correct Higgs mass (although the singlet-Higgs mixing helps). In order to be concrete, we choose to study a benchmark point $\mathrm{P} 0$, whose spectrum (as generated by NMSSMTOOLS 4.9.2 [32,33]) is shown in Fig. 2. P0 has a SM-like Higgs in the vicinity of the measured mass at $125 \mathrm{GeV}$ (allowing for a $3 \mathrm{GeV}$ theoretical uncertainty in its prediction) and a lighter CP-even Higgs at $90 \mathrm{GeV}$ that couples with a reduced strength (compared to a SM Higgs) to $Z$-bosons, commensurate with a $2 \sigma$ LEP excess. In addition, the lightest singlet-like pseudo-scalar $a_{1}$ has a mass of $23 \mathrm{GeV}$ and the singlino-like NLSP $\tilde{N}_{1}$ has a mass of $98 \mathrm{GeV}$.

We generate event samples with PYTHIA 8.2 [35], using FASTJET 3.1.3 [36] for jet reconstruction. The ATLAS models we wish to validate, described in Sect. 4.1, are generated with SOFTSUSY 3.6.1 [37,38] to calculate the spectra and SDECAY 1.5 [39] to generate the decays, communicating the spectrum and decay information via SUSY Les Houches Accord (SLHA) files [40,41].

To take into account the size of the detector, we consider a cylinder with radius $r=11 \mathrm{~m}$ and length $|z|=28 \mathrm{~m}$, corresponding to the ATLAS inner detector [42]. It is possible for a neutral particle that decays outside the inner detector to form trackless jets. However, it is difficult to model the detector response to these and so we consider them to be beyond the scope of this study. Any particle that decays outside the inner detector is therefore considered to be stable for all intents and purposes. The detector response for measurement of jet $p_{T}$ is modelled as follows. ${ }^{2}$ The jet momentum is smeared by a gaussian with resolution of $20 \%$ of energy for $E_{\text {jet }}<50 \mathrm{GeV}$, falling linearly to $10 \%$ up to $100 \mathrm{GeV}$ and then a flat $10 \%$. A further scale correction of $1 \%$ is applied for jets with $|\eta|<2$ and $3 \%$ for those with higher $|\eta|$.

With this parameterisation, we are able reproduce the cut flows for the ATLAS 0-lepton + jets + missing transverse energy $^{3}\left(p_{T}^{\text {miss }}\right)$ analyses and the efficiencies are validated against published results for benchmarks provided in the ATLAS analysis documentation. Further fiducial and material cuts required for tracks in the DV studies are explained in Sect. 4.1.

\section{Prompt SUSY searches}

In order to determine constraints on the gluino mass in our model, we focus on the 0-lepton + two-six jets + $p_{T}^{\text {miss }}$ search $[43,44]$ which is the most sensitive search for benchmark P0. However, to investigate the response of our model to dedicated SUSY searches, we deform it by moving on a line into the phenomenological next-tominimal model space (pNMSSM): for instance, we vary the gluino mass soft parameter $M_{3}$ while keeping all other weak-scale parameters fixed. The spectrum, decays and lifetimes are recomputed at each point: to first order, only the gluino mass changes, but there are small loop-level effects on other masses. Since this deformation breaks the gaugemediated relation between the gaugino masses, we are deviating from the gauge-mediated limit by doing this. This is a simple choice where we can change only one parameter; we could have equally made a different choice where we vary several weak-scale parameters-trying to preserve some of the gauge-mediated relations. Keeping within the NMGMSB model itself was not an option however, since a highly non-trivial multi-dimensional manipulation of parameters was required, which ended in some other phenomenological bound being violated. Our approach is mainly phenomenologically motivated, essentially to study the gluino mass bounds in the context of the very peculiar structure of singlino-like NLSP and gravitino LSP (with squarks decoupled). Nevertheless one might imagine a possible extension of the DGS scenario with additional sources for the Higgs

\footnotetext{
2 We find inconsistent results from standard detector simulation programs leading us to believe that the presence of DVs interferes with the standard reconstruction.

${ }^{3}$ We prefer to use the more accurate descriptor $p_{T}^{\text {miss }}=\left|\mathbf{p}_{T}^{\text {miss }}\right|$ than the ' $E_{T}^{\text {miss }}$ ' quoted by the ATLAS analyses referred to in this study.
} 
Table 1 The cuts for more sensitive signal regions from the 0-lepton + jets $+p_{T}^{\text {miss }}$ searches at $8 \mathrm{TeV}[43]$ and $13 \mathrm{TeV}$ [44] runs and $95 \%$ observed upper limits on a non-standard model contribution $\sigma_{95}^{\text {obs }}$. The limit $\sigma_{95}^{\text {obs }}$ has not been unfolded, and so should be applied to the production cross section times branching ratio times acceptance. The jets $j$ are ordered in decreasing $p_{T}$. The effective mass, $m_{\text {eff }}$ (incl.) is defined to be the scalar sum of $p_{T}$ 's of all jets with $p_{T}>40(50) \mathrm{GeV}$ for $\sqrt{s}=8$ (13) $\mathrm{TeV}$ plus the missing transverse momentum $p_{T}^{\text {miss }}, m_{\mathrm{eff}}\left(N_{j}\right)$ is the scalar sum of $p_{T}$ 's of $N_{j}$ hardest jets $\left(N_{j}=4\right.$ for $4 \mathrm{jt}-\mathrm{X}$ and $N_{j}=6$ for $6 j t-X)$ plus $p_{T}^{\text {miss }}$ and $\phi$ is the azimuthal angle around the beam

\begin{tabular}{|c|c|c|c|c|}
\hline \multirow{2}{*}{$\begin{array}{l}\sqrt{s} \\
\text { Signal region }\end{array}$} & \multicolumn{2}{|l|}{$8 \mathrm{TeV}$} & \multicolumn{2}{|l|}{$13 \mathrm{TeV}$} \\
\hline & $4 j t-8$ & $6 j t-8$ & $4 j t-13$ & $6 j t-13$ \\
\hline$p_{T}^{\text {miss }} / \mathrm{GeV}>$ & 160 & 160 & 200 & 200 \\
\hline$p_{T}\left(j_{1}\right) / \mathrm{GeV}>$ & 130 & 130 & 200 & 200 \\
\hline$p_{T}\left(j_{2}\right) / \mathrm{GeV}>$ & 60 & 60 & 100 & 100 \\
\hline$p_{T}\left(j_{3}\right) / \mathrm{GeV}>$ & 60 & 60 & 100 & 100 \\
\hline$p_{T}\left(j_{4}\right) / \mathrm{GeV}>$ & 60 & 60 & 100 & 100 \\
\hline$p_{T}\left(j_{5}\right) / \mathrm{GeV}>$ & - & 60 & - & 50 \\
\hline$p_{T}\left(j_{6}\right) / \mathrm{GeV}>$ & - & 60 & - & 50 \\
\hline$\Delta \phi\left(\text { jet }_{1,2,3}, \mathbf{p}_{T}^{\text {miss }}\right)_{\min }>$ & 0.4 & & & \\
\hline$\Delta \phi\left(\text { jet }_{j>3}, \mathbf{p}_{T}^{\text {miss }}\right)_{\min }>$ & 0.2 & & & \\
\hline$p_{T}^{\text {miss }} / m_{\text {eff }}\left(N_{j}\right)>$ & 0.25 & & 0.2 & \\
\hline$m_{\text {eff }}($ incl. $) / \mathrm{GeV}>$ & 2200 & 1500 & 2200 & 2000 \\
\hline$\sigma_{95}^{\mathrm{obs}}(\mathrm{fb})$ & 0.15 & 0.32 & 2.7 & 1.6 \\
\hline
\end{tabular}

mass that allow one to lower the overall scale of sparticle masses to the investigated range.

We have also sometimes, for the purposes of illustration only, changed the singlino decay length $c \tau_{\tilde{N}_{1}}$ (while keeping all weak-scale parameters fixed). This deformation does not really constitute a consistent model, but is used instead to understand some features that are present in consistent models. When using this type of deformation we will refer to 'tweaked' parameters. We shall investigate the effect of varying the lifetime by scanning over a lifetime range of $c \tau=\left[10^{-3}, 10^{4}\right] \mathrm{mm}$ for $m_{\tilde{g}} \sim 1 \mathrm{TeV}$.

\subsection{Current bounds from Run I and early Run II searches}

In the NMGMSB model under study, the squarks (including the third generation squarks) are usually heavier than the gluino, resulting in three-body decays through off-shell squarks of the form $\tilde{g} \rightarrow q \bar{q} \tilde{N}_{1}$, where $\tilde{N}_{1}$ is mostly singlino-like, followed by the potentially displaced decay $\tilde{N}_{1} \rightarrow \tilde{G} a_{1} \rightarrow \tilde{G} b \bar{b}$. Although the last step in the decay chain always ensures the presence of $b$ 's in the final state, they are usually too soft to satisfy the requirements of current $b$-jet $+p_{T}^{\text {miss }}$ searches. We find that $b$-jet searches only become sensitive when the mass of the gluino is high enough that decays into third generation squarks dominate and their decays into top/bottom quarks (see an example

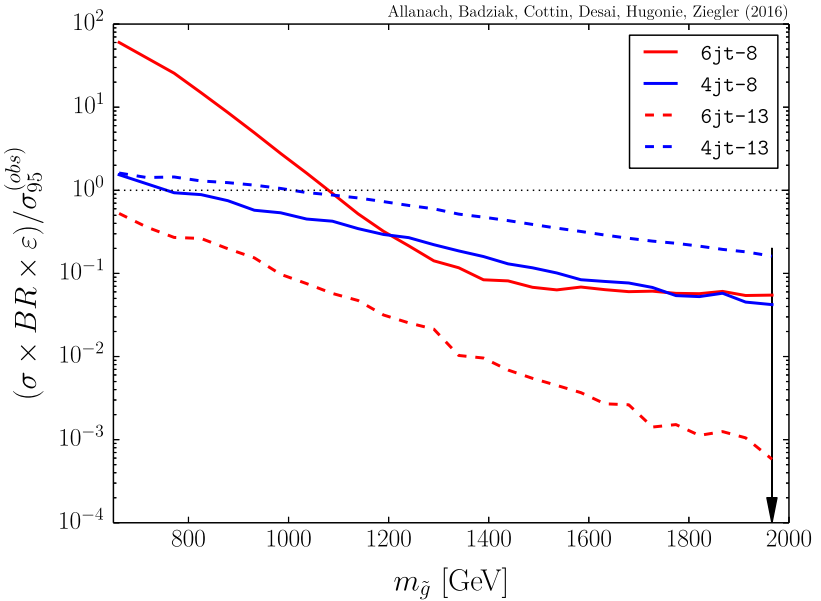

Fig. $395 \%$ lower limits on the gluino mass from Run I and Run II jets $+p_{T}^{\text {miss }}$ searches. We show the ratio of the predicted gluino cross section times branching ratio times acceptance $(\sigma \times B R \times \varepsilon)$ to the $95 \%$ upper bound on signal cross sections determined by ATLAS, for a scan-line based on benchmark $\mathrm{P} 0\left(\tilde{N}_{1}\right.$ lifetime of $\left.c \tau_{\tilde{N}_{1}}=99 \mathrm{~mm}\right)$. The horizontal dotted line shows the exclusion limit at $r=(\sigma \times B R \times \varepsilon) / \sigma_{95}^{\text {obs }}=1$. The arrow shows the position of our benchmark P0 in NMGMSB, whereas elsewhere we are strictly in pNMSSM parameter space

event topology in Fig. 1) result in high- $p_{T} b$-jets. Therefore these searches are never relevant for our benchmark $\mathrm{P} 0$ and the corresponding pNMSSM line that has even lower gluino masses. Note, however, that even when the gluino mass is high, the gluino branching fraction into $b$ 's is still only about $20 \%$ and is often accompanied by vector bosons in the final state. These sometimes produce leptons, which take events out of the 0 -lepton + multi-jets $+p_{T}^{\text {miss }}$ selection. As a result of the above considerations, the sensitivity is much lower than that from simplified models producing hard $b$-jets and missing transverse momentum. We find that the sensitivity in the simplest 0 -lepton + jets $+p_{T}^{\text {miss }}$ searches is greater than that of searches involving $b$ s even at high gluino masses. The signal regions (i.e. the labelled sets of cuts) defined by ATLAS that have the highest sensitivity are the $4 j t-8$ and $6 j t-8$ signal regions (relevant for $8 \mathrm{TeV}$ collisions) and the $4 j t-13$ and $6 j t-13$ signal regions (relevant for $13 \mathrm{TeV}$ collisions). We reproduce the cuts in these signal regions in Table 1 along with the observed upper limits on production cross section at the $95 \%$ confidence level (CL).

Since in the DGS model the gluino sets the overall mass scale of the sparticle spectrum, we therefore present our results in the form of bounds on the gluino mass. We define the signal strength ratio $r_{95}$ as the ratio of the predicted sparticle signal passing the selection cuts in a particular signal region to the $95 \% \mathrm{CL}$ upper limit on the cross section in that region. Thus $r_{95}=1$ is just ruled out to $95 \% \mathrm{CL}, r_{95}>1$ is ruled out whereas $r_{95}<1$ is allowed at the $95 \% \mathrm{CL}$. The signal region is always chosen to be the one giving the best expected exclusion. Figure 3 shows the signal strength 


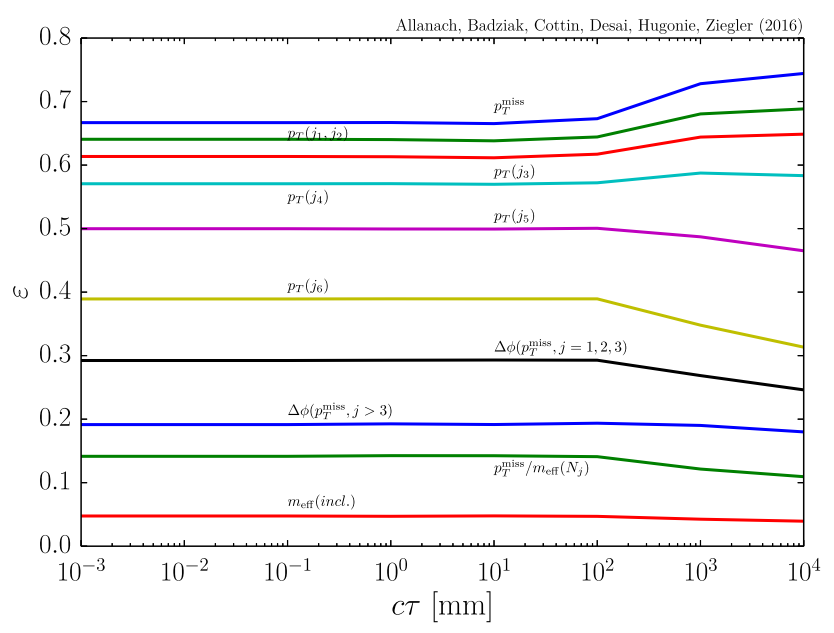

Fig. 4 The dependence of overall efficiency on lifetime in the signal region $6 j t-8$. We find that the strong dependence on $p_{T}^{\text {miss }}$ is strongly anti-correlated with the cuts on jet $p_{T}$, resulting in a fairly small dependence of the efficiency on $c \tau$ after all cuts. The curves correspond from top to bottom to the cuts in Table 1 and the corresponding variables are shown in the plot

ratio for varying gluino mass based on the pNMSSM line described in Sect. 3. The most sensitive signal region for gluinos from 0.9 to $1.2 \mathrm{TeV}$ is the $6 j t-8$ region (six hard jets, tight cuts) at $\sqrt{s}=8 \mathrm{TeV}$. For higher gluino masses up to $2 \mathrm{TeV}$, the sensitivities of the $6 j t-8$ and $4 j t-8$ signal regions are similar. The presence of a long-lived singlino which may decay within the detector leads to another possible signature - that of DVs, which we shall explore in the next section. We see from the figure that the bound from Run I at $95 \%$ is $m_{\tilde{g}}>1080 \mathrm{GeV}$, where the $6 j t-8$ line intersects $r_{95}=1$.

In 2015, ATLAS analysed $3.2 \mathrm{fb}^{-1}$ of integrated luminosity collected at the higher centre of mass energy of $13 \mathrm{TeV}$, not observing any significant signal for sparticle production. We see that at $13 \mathrm{TeV}$, the $4 j t-13$ cuts are more sensitive to our pNMSSM model than the $6 j t-13$ cuts for any value of the gluino mass, mainly because the $p_{T}$ requirements are not satisfied by the jets from singlino decay products (which give $N_{\text {jets }}>4$ ). We see that the early Run II data from 2015 constrained $m_{\tilde{g}}>1000 \mathrm{GeV}$, not as sensitive as the Run I limit. Later, we shall examine the expected sensitivity from Run II with $100 \mathrm{fb}^{-1}$ of integrated luminosity.

In Fig. 4 , we show how changing the lifetime of the $\tilde{N}_{1}$ affects the cut acceptances (shown here for the signal region $6 j t-8)$. The lines should be read in one-to-one correspondence from top to bottom with the cuts listed in Table 1 (except the second line which is the combined efficiency for $p_{T}\left(j_{1}\right)$ and $\left.p_{T}\left(j_{2}\right)\right)$. Thus, the first line corresponds to the cut $p_{T}^{\text {miss }}>160 \mathrm{GeV}$ and the last to $m_{\text {eff }}>1500 \mathrm{GeV}$. As expected, when the singlino is stable, we find large missing energy as all the momentum it carries is invisible. As the

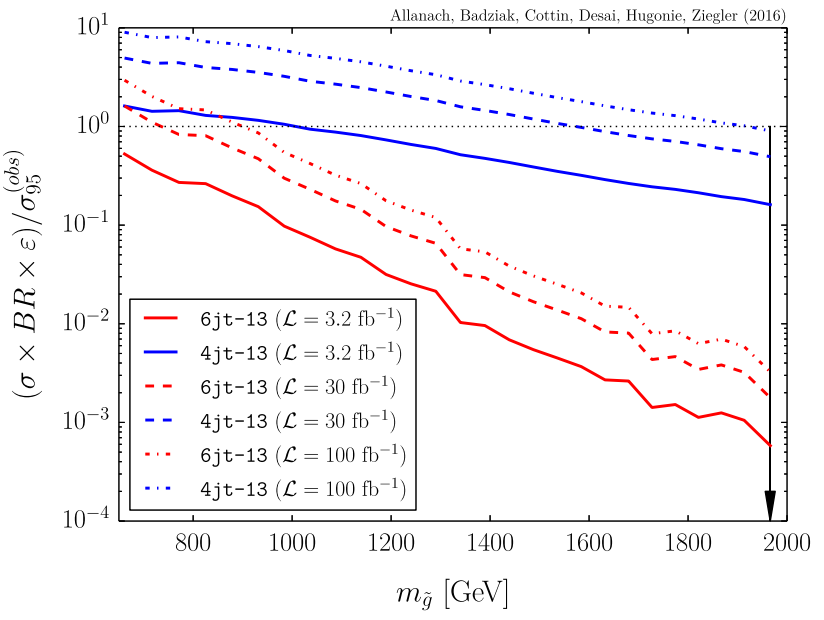

Fig. $595 \%$ CL limits the gluino mass from the $\sqrt{s}=13 \mathrm{TeV}$ jets + $p_{T}^{\text {miss }}$ searches and projected sensitivity with higher luminosity in the pNMSSM model. The signal regions $4 j t-13$ and $6 j-13$ are defined in Table 1. The arrow shows the position of our benchmark P0 in NMGMSB, whereas elsewhere we are strictly in pNMSSM parameter space

lifetime decreases, more and more singlinos decay within the detector volume resulting in a flat efficiency below lifetimes of $10 \mathrm{~mm}$. This gain in efficiency for long-lived $\tilde{N}_{1}$ is somewhat diluted once we demand jets with high $p_{T}$. In particular, once we demand $N_{\text {jets }}>4$, the efficiency is lower for stable singlinos, since the extra hard jets mainly come from decay products of the $\tilde{N}_{1}$. However, this downturn is balanced by the requirements on $\Delta \phi$ between the jets and the missing momentum $p_{T}^{\text {miss }}$, since the presence of more jets in the final state makes it harder to satisfy this cut. Finally, after all cuts, we find that the efficiency is rather flat across all lifetimes. Therefore the gluino mass limits presented above may be considered fairly robust for the model studied here.

\subsection{Future search reach of prompt searches}

We now estimate what the future might bring for discovery or exclusion of the pNMSSM model from the LHC. In Fig. 5, we re-display the current limits on the gluino in PNMSSM from the $13 \mathrm{TeV}$ run, as the gluino mass is changed for the $6 j t-13$ and $4 j t-13$ signal regions. The solid lines show the current lower limit from the 2015 run of $1000 \mathrm{GeV}$. For our model, the $4 j t-13$ region performs better than the $6 j t-13$ region. Model sensitivity (ignoring systematic errors) is equal to the number of signals events $S$ divided by the square root of the number of background events $B$. Since $S \propto$ the total integrated luminosity $\mathcal{L}$, and $B \propto \mathcal{L}$, the sensitivity scales $\propto \sqrt{\mathcal{L}}$. Thus, we expect $\sigma_{95}^{\text {obs }} \propto 1 / \sqrt{\mathcal{L}}$. Using this dependence, we scale the $\mathcal{L}=3.2 \mathrm{fb}^{-1}$ lines to 30 and $100 \mathrm{fb}^{-1}$ to show the projected sensitivities in the figure. We see that 
with $100 \mathrm{fb}^{-1}$ and $13 \mathrm{TeV}$ centre of mass collision energy, the LHC can reach up to $1900 \mathrm{GeV}$ gluinos.

\section{Searches with displaced vertices}

DV searches are especially challenging due to the complication of taking into account time of flight and assigning tracks originating far away from the primary interaction point to the correct event. Reconstruction of such decays therefore becomes more difficult beyond the pixel layers. Nevertheless, these searches have an extremely low background as there are no irreducible contributions from the SM. Some recent reinterpretation of LHC displaced searches can be found in Refs. [45-51]. Displaced signatures have received far less attention in the literature as compared to prompt signatures because they are difficult to model, and because they tend to be rather model specific. Furthermore, modelling the detector's response to DVs is a difficult task, as we shall illustrate. Validation is therefore essential in order to tell how good or bad a job of modelling the response we achieve. References $[45,47]$ used truth information to identify displaced decays. Our work goes further by fully detailing the steps of reconstruction for DVs, in a similar way to Ref. [46], but here we determine an explicit functional form for the tracking efficiency, which is needed to be able to model the efficiencies from the experiments to a reasonable (if somewhat rough) level.

\subsection{Validation of Run I displaced vertex searches}

In the absence of publicly available multi-dimensional, model-independent efficiency maps for the reconstruction efficiency of a DV, we make use of the efficiencies published for specific models and construct a function that approximately simultaneously reproduces them. The ATLAS DV + jets search [52] has been interpreted in the context of two General Gauge Mediation (GGM) and several $R$-parity violating supersymmetry (RPV) simplified models. Of these, the ones most relevant to signatures predicted by the DGS model (where we expect only jets from the DV) are the two GGM model benchmarks and one RPV benchmark where a displaced neutralino decays through a non-zero $\lambda_{211}^{\prime}$ to light quarks and a muon.

The ATLAS DV + jets cuts are summarised in Table 2 . The ATLAS analysis re-runs the experiment's standard tracking algorithms on events passing the trigger in order to determine the efficiency for the displaced tracks. Given the fact that we do not have access to such algorithms, we assign each track a reconstruction probability depending on its $p_{T}$ and the true co-ordinates of its displaced origin. The functional form found to reproduce the efficiencies for the three benchmark models is given by
Table 2 Our implementation of cuts applied in the ATLAS multi-track $\mathrm{DV}+$ jets search, from Ref. [52]

\begin{tabular}{lc}
\hline DV jets & 4 or 5 or 6 jets with $|\eta|<2.8$ and \\
& $p_{T}>90,65,55 \mathrm{GeV}$, each \\
DV reconstruction & DV made from tracks with $p_{T}>1 \mathrm{GeV}$, \\
& $|\eta|<2.5$ and $\left|d_{0}\right|>2 \mathrm{~mm}$, satisfying a \\
& tracking efficiency given by Eq. 2. \\
& Vertices within $1 \mathrm{~mm}$ are merged \\
& DV within $4 \mathrm{~mm}<r_{\mathrm{DV}}<300 \mathrm{~mm}$ and \\
DV fiducial & $\left|z_{\mathrm{DV}}\right|<300 \mathrm{~mm}$ \\
& No DV in regions near beampipe or \\
DV material & within pixel layers: \\
& Discard tracks with $r_{\mathrm{DV}} / \mathrm{mm} \in$ \\
& $\{[25,38],[45,60],[85,95],[120,130]\}$ \\
$N_{\text {trk }}$ & DV track multiplicity $\geq 5$ \\
$m_{\mathrm{DV}}$ & DV mass $>10 \mathrm{GeV}$ \\
\hline
\end{tabular}

$$
\begin{aligned}
\varepsilon_{\mathrm{trk}}= & 0.5 \times\left(1-\exp \left(-p_{T} /[4.0 \mathrm{GeV}]\right)\right) \\
& \times \exp (-|z| /[270 \mathrm{~mm}]) \\
& \times \max \left(-0.0022 \times r_{\perp} /[1 \mathrm{~mm}]+0.8,0\right),
\end{aligned}
$$

where $r_{\perp}$ and $z$ are the transverse and longitudinal distance of the track's production vertex (for details of their definitions, see Appendix A).

We pick this particular parameterisation of the tracking efficiency after trying several functional forms and varying the constants, picking the one that had the best goodness of fit statistic $\left(\chi^{2}\right)$ for the three models combined that we validate against (at various different values of lifetimes of the decaying sparticle). Equation (2) is not expected to be perfect by any stretch: it is a simple, universal and factorised form for the track efficiency that is a rough approximation. The overall $\chi^{2}$ statistic did not indicate a particularly good fit; however, inspection by eye showed that the shapes of the efficiency curves were reasonable. We display contours of the function in Fig. 6.

The efficiency for reconstructing a multi-track DV is highly dependent on track reconstruction and track selection, as detailed in Ref. [52]. These are affected by several factors:

- The impact parameter $d_{0}$ of the track: the efficiency for reconstructing tracks decreases with increasing values of $d_{0}$, since the density of fine instrumentation decreases.

- The mass of the long-lived particle: as the number of tracks originating from the vertex increases with increasing mass. At higher masses, missing some tracks may therefore still lead to the identification of a DV.

- The energy of the long-lived particle: the higher the boost, the more tracks will have a small angle with respect to the flight direction of the long-lived particle and may therefore fail the minimal $d_{0}$ cut. 
Fig. 6 Contours of track efficiency as a function of $r_{\perp}$ and $|z|$, for a $p_{T}$ value fixed at $1 \mathrm{GeV}$ (left) and $10 \mathrm{GeV}$ (right). Note the difference scales of track efficiency (labelled by the legend at the right-hand side) of each panel

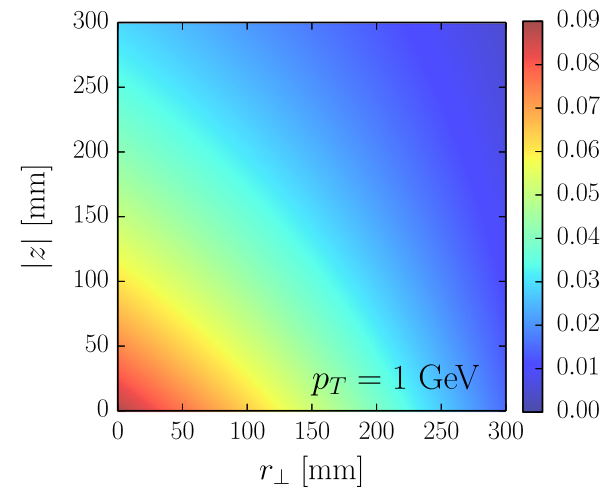

We can therefore see that the vertex reconstruction efficiency will be a non-trivial combination of different aspects of the track reconstruction efficiency. For instance the vertex reconstruction efficiency is worst at large radii, which is due to tracking efficiency decreasing. Here, we focus only on assigning each track a reconstruction probability dependent on some relevant variables, such as the transverse and longitudinal distance of the production vertex of the track and its transverse momentum. The particular choice of the variables in Eq. 2 (for instance the fact we use $r_{\perp}$ instead of $d_{0}$ ) was made because we found these fitted the best across the three different signal models out of a few different simple factorised functional forms that we tried.

We find that validating against only one of the benchmark models at a time leads to different best-fit parameters for each. Using three benchmarks for validation therefore gives us more confidence in applying the efficiency to our own model. We believe this is a key improvement in our work. Choosing a functional form for the tracking efficiency in order to recast displaced results has already been attempted in the literature [46]. Here we show the explicit functional form used, since knowing it is necessary to be able to reproduce our results. Figure 7 shows the validation of our simulation (dashed lines) for three different ATLAS benchmarks, against the ATLAS determination (solid lines). We see that the efficiency, while far from being perfectly modelled by our function, is adequately modelled (within a few sigma) for most of the range of lifetimes considered. We could improve the above fits by including an additional selection efficiency at the vertex level, as discussed above. We could also take into account the topology of the different signatures. Ideally, a parameterisation of the tracking efficiency should be validated against all the $\sim 20$ signal benchmarks used in the ATLAS search, which is beyond the scope of this paper and which we leave for a future work.

Undiscarded displaced tracks are input into our vertex reconstruction algorithm, which compares and clusters the tracks' origins. ${ }^{4}$ If the origins of two displaced tracks are less than $1 \mathrm{~mm}$ apart, then they are clustered together into one DV. Picking the first track, we compute the $d$ value (i.e. the physical distance in the laboratory frame $\sqrt{\Delta x^{2}+\Delta y^{2}+\Delta z^{2}}$ ) to each of the other tracks, clustering tracks that have a small enough $d$ value to the first track. Then we repeat for the next unclustered track and so on, until each track is assigned to a single vertex. The ATLAS analysis (and ours) combines vertices into a DV if they are less than $d=1 \mathrm{~mm}$ apart. The DV position is defined as the average position of all the track origins in the cluster.

To ensure consistency of the vertex position and the direction of the tracks, we require at least two tracks in the vertex to have $\mathbf{d} \cdot \mathbf{p}>-20 \mathrm{~mm}$, where we define $\mathbf{d}$ to be the vector from the interaction point to the DV and $\mathbf{p}$ to be the momentum of the displaced track. In the ATLAS analysis, DVs are vetoed if they are reconstructed in high density material regions, since this is the main source of background vertices. We simulate this by requiring $4 \mathrm{~mm}<r<300 \mathrm{~mm}$ and $|z|<300 \mathrm{~mm}$. We also require decay positions of the DVs to not be inside any of the three ATLAS pixel layers (our approximation to this DV material cut is shown in Table 2). ${ }^{5}$ As the table shows, events are further selected if they have at least one reconstructed DV with five tracks or more and a DV invariant mass (computed assuming all tracks have the pion mass) of at least $10 \mathrm{GeV}$.

\subsection{Run I sensitivity of displaced vertex searches}

We now apply the simulation described in the previous section to the DGS model benchmark P0. We find that the sensitivity of the ATLAS study to our benchmark is extremely

\footnotetext{
${ }^{4}$ ATLAS performs a complicated vertex $\chi^{2}$ fit in order to reconstruct DVs. Here, we simply use the truth information to define the track's origin to be the point at which the $\tilde{N}_{1}$ decays, and start comparing the distance between tracks' origins to cluster them into vertices.

5 Note that the material veto performed in the ATLAS analysis is far more complex than this, since ATLAS makes use of a 3D material map of the detector that we do not have access to.
} 
Fig. 7 Validation of our DV + jets estimate of efficiency for three models against ATLAS's determination: a a simplified GGM model where a $1.1 \mathrm{TeV}$ gluino decays to a $400 \mathrm{GeV}$ neutralino, which in turn decays to a $Z$ and a gravitino, $\mathbf{b}$ a simplified GGM model where a 1.1 TeV gluino decays to a $1 \mathrm{TeV}$ neutralino, which in turn decays to a $Z$ and a gravitino, c a simplified RPV model with a $700 \mathrm{GeV}$ squarks decaying to a $500 \mathrm{GeV}$ neutralino, which subsequently decays through a non-zero $\lambda_{211}^{\prime}$ coupling into a muon and two quarks. Events are generated with $\sqrt{s}=8 \mathrm{TeV}$. The bottom rectangle in each case shows the discrepancy between our estimate and ATLAS's, measured in units of the ATLAS error

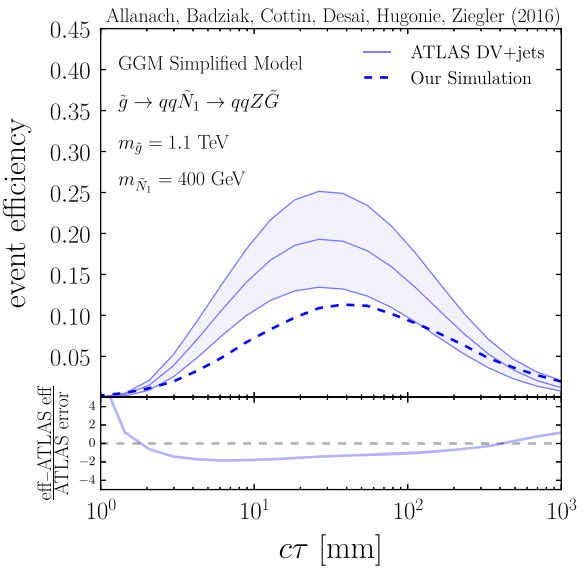

(a) GGM1



(b) GGM2

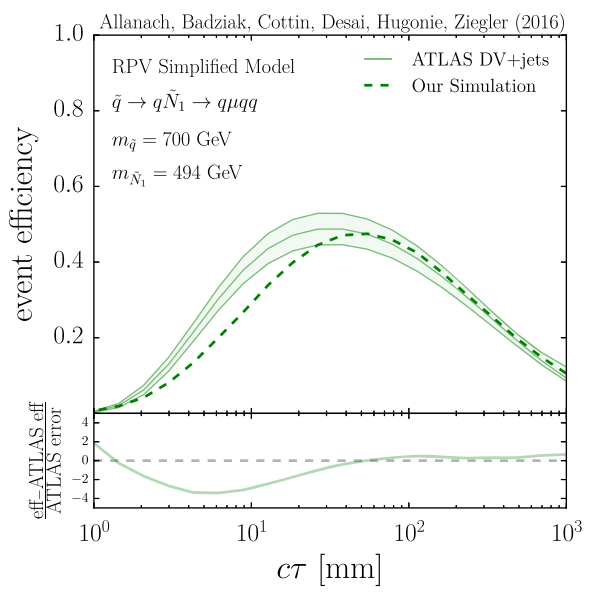

(c) RPV

Table 3 Numbers of simulated events $N$ and relative efficiencies $\epsilon$ (i.e. defined with respect to the previous cut) for our NMGMSB model (P0 benchmark) with $c \tau_{\tilde{N}_{1}}=99 \mathrm{~mm}$ at $\sqrt{s}=8 \mathrm{TeV}$ and $\sqrt{s}=13 \mathrm{TeV}$ for the ATLAS selection of cuts in Table 2

\begin{tabular}{|c|c|c|c|c|}
\hline & \multicolumn{2}{|c|}{$\sqrt{s}=8 \mathrm{TeV}$} & \multicolumn{2}{|c|}{$\sqrt{s}=13 \mathrm{TeV}$} \\
\hline & $N$ & $\epsilon(\%)$ & $N$ & $\epsilon(\%)$ \\
\hline All events & 100,000 & 100.0 & 100,000 & 100.0 \\
\hline DV jets & 96,963 & 97.0 & 98,306 & 98.3 \\
\hline DV reconstruction & 16,542 & 17.1 & 16,542 & 16.8 \\
\hline DV fiducial & 16,459 & 99.5 & 16,460 & 99.5 \\
\hline DV material & 16,146 & 98.1 & 16,210 & 98.5 \\
\hline$N_{\text {trk }}$ & 584 & 3.6 & 544 & 3.4 \\
\hline$m_{\mathrm{DV}}$ & 4 & 0.7 & 3 & 0.6 \\
\hline
\end{tabular}

limited. From the $8 \mathrm{TeV}$ columns of Table 3 , it is clear that the primary cause for this is failure to satisfy the requirements $N_{\text {trk }} \geq 5$ and the vertex mass cut $m_{\text {DV }}>10 \mathrm{GeV}$. This is due to the fact that the displaced jets are mainly $b$-jets. The $b$ hadrons are themselves long-lived, and the neutral $B^{0}$ leaves no tracks before its decay. The topology of this final state then has two further DVs, each with less than five tracks. The ATLAS analysis does merge vertices (defined as having at least two tracks) that are within $1 \mathrm{~mm}$ of each other to possibly obtain a better vertex. However, the $b$-hadrons are sufficiently long-lived so that the resultant vertices are almost always more than $1 \mathrm{~mm}$ apart. ${ }^{6}$ For the benchmark P0 for instance, the average displaced track efficiency is 0.06 , and the average number of tracks coming from a displaced $b$ is 18.1 (after hadronisation, but before cuts). Thus, on average, there are only $18.1 \times 0.06=1.2$ visible tracks per displaced $b$.

A further consideration is the small mass of the $a_{1}$ which decays to $b \bar{b}$ (23 GeV for the benchmark P0) since softer $b$ quarks means less radiation, implying fewer tracks. The distribution of track multiplicity versus invariant mass is shown in Fig. 8. One can see clearly from the right panel that increasing the $a_{1}$ mass to $70 \mathrm{GeV}$ (done ad hoc for the purposes of

\footnotetext{
6 The ATLAS analysis [52] also reports that the sensitivity is severely reduced if they use the RPV benchmark with $b$-quarks in the final state. An earlier work on displaced Higgs decays [48], also shows how displaced $b$-quarks can be problematic, particularly given the $d<1 \mathrm{~mm}$ requirement for merging vertices.
} 
Fig. 8 DV invariant mass against number of tracks for the DGS benchmark P0 with $m_{\tilde{g}}=1.96 \mathrm{TeV}, m_{\tilde{N}_{1}}=98 \mathrm{GeV}$ and $c \tau_{\tilde{N}_{1}}=99 \mathrm{~mm}$. Gluinos and squarks only are generated with $\sqrt{s}=8 \mathrm{TeV}$. Events in the plot pass all of the DV cuts except for the last two, which define the boxed ATLAS signal region. The left-hand frame shows a scenario where $m_{a_{1}}=23 \mathrm{GeV}$ (in the DGS good-fit region, as in $\mathrm{P} 0$ ) and the right-hand frame has a tweaked $m_{a_{1}}=70 \mathrm{GeV}$ in the SLHA file (i.e. inconsistent with the soft parameters, which are left constant-for the purposes of illustration only)
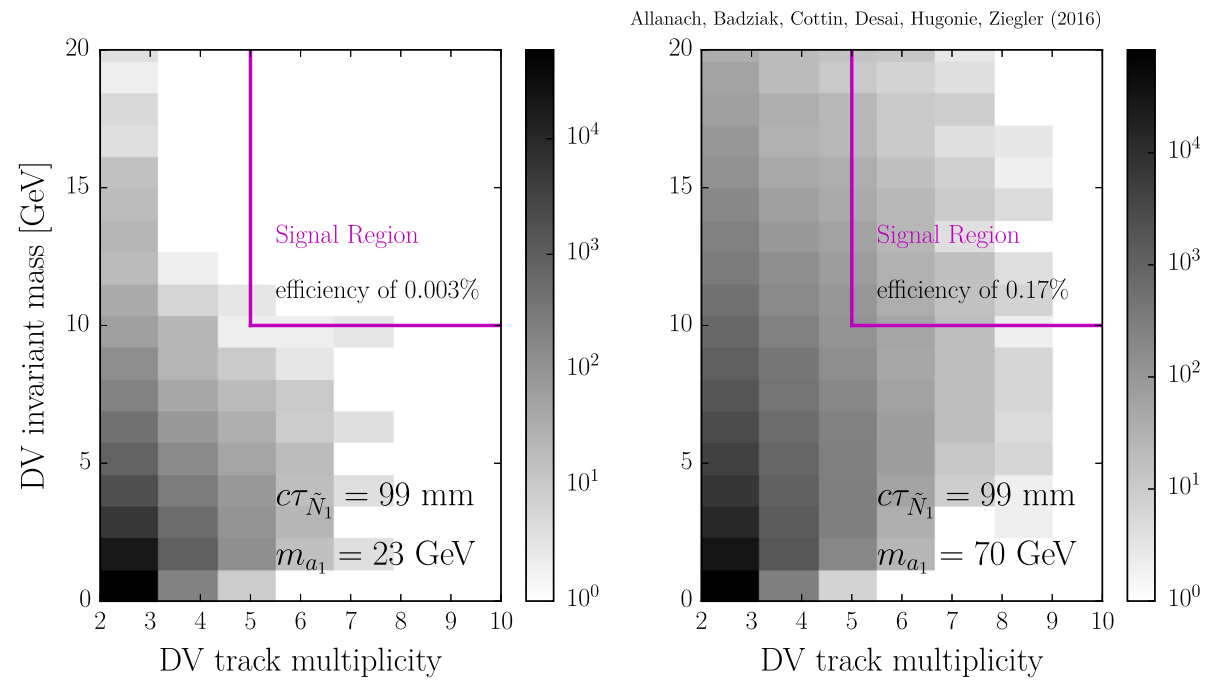

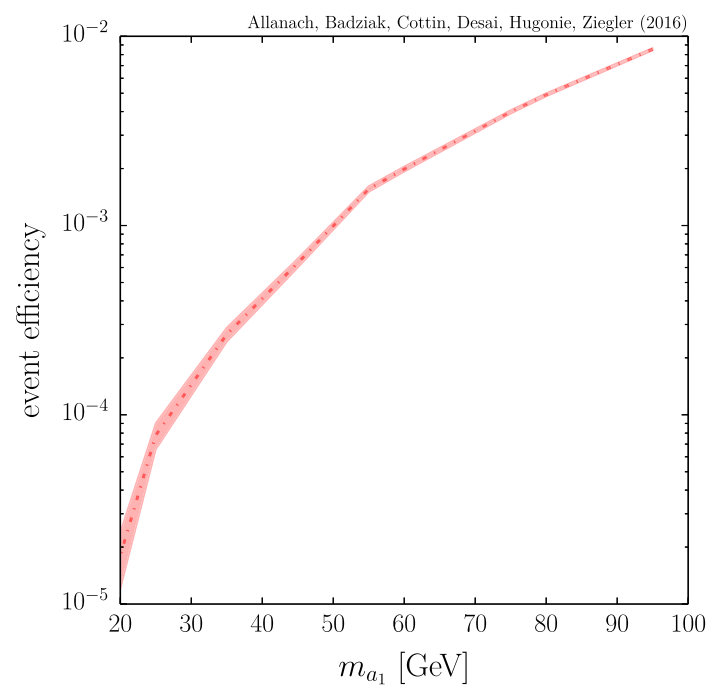

Fig. 9 Event efficiency against pseudo-scalar mass for a DGS benchmark with $c \tau_{\tilde{N}_{1}}=99 \mathrm{~mm}$ (our P0 benchmark). Events are generated with $\sqrt{s}=8 \mathrm{TeV}$ considering strong production. We have tweaked $m_{a_{1}}$ "by hand" in the SLHA files without changing soft parameters for the purposes of illustration

illustration) improves the sensitivity of the cuts by two orders of magnitude. A higher mass also means the resultant products are more collimated and hence the $b$-hadron vertices are likely to be closer to each other. The improvement in efficiency with increasing $a_{1}$ mass can be seen in Fig. 9 .

\subsection{Improving the sensitivity of displaced vertex searches}

Given the very low sensitivity of the DV searches, we shall now attempt to improve it by loosening the most restrictive cuts. Firstly, to catch DVs coming from two $b$-quarks from the same $a_{1}$, we relax the requirement of maximum merging distance from 1 to $5 \mathrm{~mm}$. Further, we can also relax the last two cuts: track multiplicity and invariant mass of the DV.

The background to the DV multi-track search comes from three sources-heavy flavour quark decays, interactions with material in the detector and the accidental crossing of tracks, all of which have a low multiplicity of tracks and a small invariant mass of the DV. Thus, if we loosen these cuts to achieve better signal efficiency, we also raise the background rate thus reducing the signal to background ratio. However, given that our model has good sensitivity in the prompt $p_{T}^{\text {miss }}$ based channels, background rates can be controlled by taking advantage of the hard prompt signals that come in association with the DVs. Requiring a large $m_{\text {eff }}$ in the event would reduce backgrounds significantly. It may also be possible to increase the sensitivity by loosening the DV cuts but requiring displaced jets to have a muon inside them [53,54] (which often come from a $b$ ). However, we do not consider this route here.

We now investigate the effect of applying prompt cuts used in standard jets $+p_{T}^{\text {miss }}$ sparticle searches on top of relaxed DV cuts. This, of course, will have a lower signal efficiency than purely applying the standard jets $+p_{T}^{\text {miss }}$ cuts, which are already designed to remove the SM background very effectively. Ideally, one would optimise the jets $+p_{T}^{\text {miss }}$ cuts along with the DV cuts to reach an overall best sensitivity. However, we have clear estimates of the background to the prompt channels from the analysis which serves as an upper bound to any DV contributions we may have from heavy flavour. Of course, the contributions from systematic sources cannot be bounded in this way, however, we can reasonably assume that the number of DVs from systematic sources is not biased by the hard cuts we place.

At $8 \mathrm{TeV}$, we choose the ATLAS $6 j t-8$ signal region cuts described in Table 1, because they were found to have the highest sensitivity to our signal, as shown above. Figure 10 


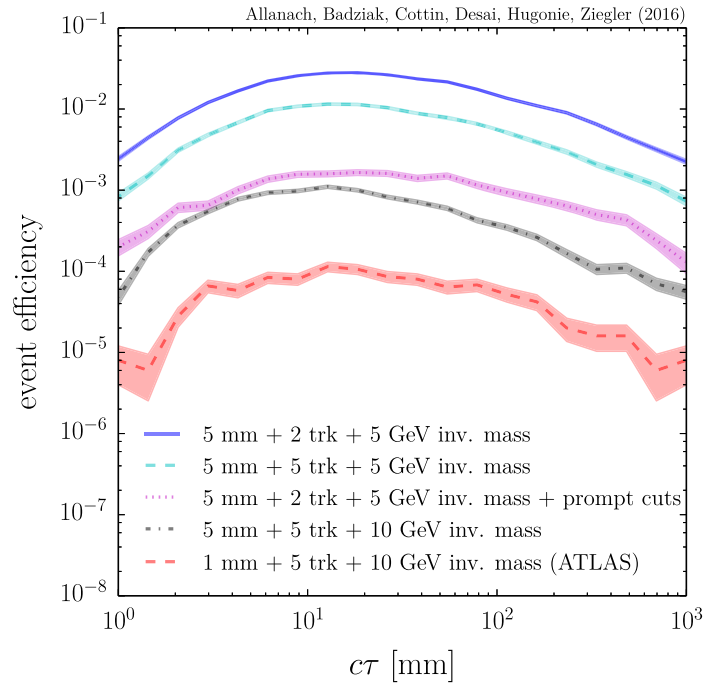

Fig. 10 Signal efficiencies of different sets of DV + jets analyses on a DGS benchmark with $m_{\tilde{g}}=1.96 \mathrm{TeV}, m_{\tilde{N}_{1}}=98 \mathrm{GeV}, m_{a_{1}}=23 \mathrm{GeV}$ (our P0 benchamark) against the lifetime of the long-lived singlino $c \tau_{\tilde{N}_{1}}$ (changed "by hand" in the SLHA files without changing the soft terms for the purposes of illustration). Events are generated with $\sqrt{s}=$ $8 \mathrm{TeV}$, considering gluino/squark production only. The bottom curve corresponds to the efficiency after the default ATLAS DV cuts. The top curve corresponds to the loosest of our selections in DV merging distance, track multiplicity and invariant mass. We also show different sets of cuts in between, including the inclusion of standard prompt cuts, as defined in the text

shows efficiency curves against lifetime for the NMGMSB model with the default ATLAS DV analysis cuts and some choices of relaxed cuts. This includes (1) allowing $N_{\text {trk }}$ to be $\geq 2$ rather than $\geq 5$, (2) increasing the vertex merging distance from 1 to $5 \mathrm{~mm}$, and (3) lowering the vertex mass cut from 10 to $5 \mathrm{GeV}$. For comparison, we also show the response for the original tight ATLAS DV (DVT) cuts as well as our loose cuts (DVL) for the $6 j t-8$ signal region. With this combination, we already achieve an improvement in signal efficiency by a factor of ten. Without the $6 j t-8$ cuts, the improvement is a factor of several hundred. An optimised analysis will be between these two limiting cases and may therefore be reasonably expected to offer an improvement of two orders of magnitude or so.

\subsection{Recommendations for displaced vertex searches at $13 \mathrm{TeV}$}

We used $6 j t-8$ for the prompt cuts at $8 \mathrm{TeV}$; however, keeping in mind that the best sensitivity at $13 \mathrm{TeV}$ is for the $4 j t-13$ signal region, we also perform efficiency calculations with the combination DVL $+4 j t-13$. The efficiencies are shown in Table 4. The signal efficiency at $13 \mathrm{TeV}$ is $\sim 0.2 \%$. It would be desirable to relax the prompt cuts further in order to increase this number, but a proper estimate
Table 4 Numbers of simulated events $N$ and relative efficiencies $\epsilon$ (i.e. defined with respect to the previous cut) for our NMGMSB model with $c \tau_{\tilde{N}_{1}}=99 \mathrm{~mm}$ (our P0 benchmark) at $\sqrt{s}=8 \mathrm{TeV}$ and $\sqrt{s}=$ $13 \mathrm{TeV}$ for our tuned cuts, as explained in the text. Events are generated considering strong production. An asterisk denotes that the prompt cuts are taken from signal regions $6 j t-8$ at $\sqrt{s}=8 \mathrm{TeV}$ and $4 j t-13$ at $\sqrt{s}=13 \mathrm{TeV}$ as listed in Table 1 . The dagger is a reminder of the increased vertex merging distance of $5 \mathrm{~mm}$

\begin{tabular}{|c|c|c|c|c|}
\hline & \multicolumn{2}{|c|}{$\sqrt{s}=8 \mathrm{TeV}$} & \multicolumn{2}{|c|}{$\sqrt{s}=13 \mathrm{TeV}$} \\
\hline & $N$ & $\epsilon(\%)$ & $N$ & $\epsilon(\%)$ \\
\hline All events & 100,000 & 100.0 & 100,000 & 100.0 \\
\hline Prompt $p_{T}^{\text {miss* }}$ & 91,709 & 91.7 & 87,737 & 87.7 \\
\hline Prompt jets* & 72,075 & 78.6 & 84,178 & 95.9 \\
\hline $\begin{array}{l}\text { Prompt } \\
\qquad \Delta \phi\left(\text { jet }_{1,2,3}, \mathbf{p}_{T}^{\text {miss }}\right)_{\min } *\end{array}$ & 49,095 & 68.1 & 57,261 & 68.0 \\
\hline $\begin{array}{l}\text { Prompt } \\
\qquad \Delta \phi\left(\text { jet }_{j>3}, \mathbf{p}_{T}^{\text {miss }}\right)_{\min } *\end{array}$ & 27,315 & 55.6 & 33,832 & 59.1 \\
\hline Prompt $p_{T}^{\text {miss }} / m_{\mathrm{eff}}\left(N_{j}\right)^{*}$ & 6670 & 24.4 & 18,409 & 54.4 \\
\hline Prompt $m_{\mathrm{eff}}$ (incl.) ${ }^{*}$ & 6636 & 99.5 & 16,848 & 91.5 \\
\hline DV jets & 6636 & 100.0 & 16,848 & 100.0 \\
\hline DV reconstruction $^{\dagger}$ & 1524 & 23.0 & 3850 & 22.9 \\
\hline DV fiducial & 1516 & 99.5 & 3825 & 99.4 \\
\hline DV material & 1494 & 98.5 & 3750 & 98.0 \\
\hline$N_{\text {trk }} \geq 2$ & 1494 & 100.0 & 3750 & 100.0 \\
\hline$m_{\mathrm{DV}}>5 \mathrm{GeV}$ & 88 & 5.9 & 265 & 7.1 \\
\hline
\end{tabular}

would require a full estimation of the DV background, which is beyond the scope of this paper.

However, an estimate of the contribution from heavy flavours may be obtained. Given the hard multi-jet, $p_{T}^{\text {miss }}$ and $m_{\mathrm{eff}}$ cuts, the dominant SM background is from $t \bar{t}+$ jets production which is also a source of $b$-hadrons and therefore a potential background for DVs. In order to examine this possibility, we simulate $10^{6} t \bar{t}$ events, and inspect the transverse impact parameter $d_{0}$ of the tracks coming from the displaced $b$ vertices. We see that only a tiny fraction of tracks pass $\left|d_{0}\right|>2 \mathrm{~mm}$ from $t \bar{t}$ events $(\sim 1 \%)$. Furthermore, imposing the DV cuts (without any restrictions on hard jets), gives us an efficiency of $0.1 \%$ for $N_{\text {trk }} \geq 2$ and imposing $m_{\mathrm{DV}}>5 \mathrm{GeV}$ gives us no events at all. We therefore do not expect any DV contributions from heavy flavour once the hard jet cuts are made. This implies zero background events at $3.2 \mathrm{fb}^{-1}$ and we are already potentially sensitive to signal cross sections of approximately $0.3 \mathrm{fb}$.

The total strong sparticle production cross section at $13 \mathrm{TeV}$ before cuts is $5.8 \mathrm{fb}$, and so with our illustrative cuts (DVL $+4 j t-13)$, one would achieve a signal cross section after cuts of $0.01 \mathrm{fb}$. With no expected background, the observation of a single event already corresponds to discovery, which for a gluino mass of $\sim 2 \mathrm{TeV}$ (as in $\mathrm{P} 0$ ) is not achievable in prompt search channels with $100 \mathrm{fb}^{-1}$ at $13 \mathrm{TeV}$. We may reasonably set the observation of at least 
three signal events as a requirement for discovery, which results in a best-case scenario of discovering a NMGMSB model with $m_{\tilde{g}} \sim 2 \mathrm{TeV}$ with $300 \mathrm{fb}^{-1}$ data at $13 \mathrm{TeV}$.

We can also make an estimate of the worst-case scenario where there is a large DV background from systematic sources. Such a background occurs when a spurious track crosses an existing DV resulting in a reconstructed vertex satisfying the $N_{\text {trk }}$ and $m_{\mathrm{DV}}$ requirements. The ATLAS DV analysis [52] estimates only $\sim 0.4$ background vertices in the $\mathrm{DV}+$ jet channel for the full $20 \mathrm{fb}^{-1}$ data of Run I (see Table 1 of Ref. [52]). Given a $t \bar{t}$ production cross section $\sim O(100 \mathrm{pb})$ at $8 \mathrm{TeV}$, this implies an efficiency $\sim 10^{-5}$. To be conservative about the effect of our relaxed cuts, we can assume that this happens in about $1 \%$ of events that pass the $4 j t-13$ cuts. Starting with a total prompt background of $\sim 1 \mathrm{fb}$ (see Table 4 of Ref. [44]) in the $4 j t-13$ channel as reported in the ATLAS analysis, we arrive at $0.01 \mathrm{fb}$ for $\mathrm{DVL}+4 j t-13$. A 3-sigma discovery may then be viable with $\sim 1 \mathrm{ab}^{-1}$ data at $13 \mathrm{TeV}$.

This situation may be improved considerably by relaxing the prompt cuts. An indication of where we may further relax the selection cuts comes from examining the relative efficiencies at 8 and $13 \mathrm{TeV}$ for the cut on the ratio of $p_{T}^{\text {miss }}$ and $m_{\text {eff }}\left(N_{j}\right)$. We see that a change from $>0.25$ at $8 \mathrm{TeV}$ to $>0.2$ at $13 \mathrm{TeV}$ (see Table 1) already results in a gain of a factor 2. Although this is obviously also due to the increased energy of the overall event, given that we have high $p_{T}^{\text {miss }}$ and $m_{\text {eff }}$ cuts, an additional factor of 3 may be gained by dropping the $p_{T}^{\text {miss }} / m_{\text {eff }}\left(N_{j}\right)$ cut altogether.

We now study how the cut efficiencies behave with singlino lifetime for benchmark P0. The result is shown in Fig. 11, where we plot the effect of the cuts DVL $+4 j t-13$ as a function of the decay length $c \tau_{\tilde{N}_{1}}$. Note that we have merged the $m_{\text {eff }}$ (incl.) and the DV jets cuts together into one curve, as applying the DV jets cut after the $m_{\text {eff }}$ (incl.) one does not change the number of events, for any lifetime (this can also be appreciated for P0 in Table 4). We notice that standard prompt cuts are not very much affected by the singlino lifetime, except for the cut on the ratio of $p_{T}^{\text {miss }}$ and $m_{\text {eff }}\left(N_{j}\right)$, which increases at higher lifetimes. This is because $p_{T}^{\text {miss }}$ is higher at high lifetimes, as explained in Sect. 3.1.

To summarise, with a combination of relaxed DV cuts and prompt SUSY search cuts, one can discover a NMGMSB scenario with $m_{\tilde{g}} \sim 2 \mathrm{TeV}$ with $300 \mathrm{fb}^{-1}$ data which is not possible with prompt SUSY searches alone. With a full optimisation of relaxed DV cuts + prompt $p_{T}^{\text {miss }}$-based cuts, we may easily gain a further factor of ten in the signal efficiency and given almost zero background, as shown above, one could have higher sensitivity to the NMGMSB model in $\mathrm{DV}+$ prompt searches as compared to prompt searches. We therefore strongly urge the experiments to perform a dedicated background simulation with optimised cuts.



Fig. 11 Signal event efficiency of our simulation on a DGS benchmark with $m_{\tilde{g}}=1.96 \mathrm{TeV}, m_{\tilde{N}_{1}}=98 \mathrm{GeV}, m_{a_{1}}=23 \mathrm{GeV}$ (our P0 benchmark) against the lifetime of the long-lived singlino $c \tau_{\tilde{N}_{1}}$. Events are generated with $\sqrt{s}=13 \mathrm{TeV}$ considering strong production. Independent prompt and DV cuts are presented. The singlino decay distance $c \tau$ has been tweaked "by hand" in the SLHA files without changing soft parameters for the purposes of illustration

\section{Summary}

We have examined the prospects for discovery or exclusion of the DGS-NMGMSB model. The model has some nice properties: the SUSY flavour problem is addressed by gaugemediated SUSY breaking, while the Higgs mass is made heavy enough through the mixing with the NMSSM CP-even singlet. This singlet has a mass around $90 \mathrm{GeV}$, and therefore can be made consistent with some small excesses in the LEP Higgs searches. An interesting feature of the model is the presence of a gravitino LSP and a singlino-like neutralino NLSP that can be long-lived.

As well as having the usual hard jets plus missing transverse momentum signatures, the model predicts possible DVs from long-lived singlinos. These decay into $b \bar{b}$ and missing transverse momentum in the form of gravitinos. However, displaced $b$ 's are somewhat problematic since $B$ mesons themselves travel a small distance before visibly decaying and the 'displaced displaced' vertices have a very poor signal efficiency for getting past the standard DV cuts. We have illustrated how loosening the DV searches whilst imposing some prompt cuts to control background results in significantly higher signal efficiency, motivating a proper study with a full detector simulation (DV analyses are difficult to perform accurately from outside the experimental collaborations). We have provided a rough approximation to the tracking efficiency that works for two General Gauge Mediation models and one $R$-parity violating model over a range of 
possible DV lifetimes, but clearly more work can be done to provide a more comprehensive parameterisation.

We have recast current $8 \mathrm{TeV}$ prompt searches to bound the gluino mass from below at $1080 \mathrm{GeV}$, whereas current $13 \mathrm{TeV}$ prompt searches are less restrictive. This is somewhat low compared to naive expectations based on LHC exclusion results quoted for simplified models, but as Fig. 2 shows, there are many different cascade decays in the model. This means that the supersymmetric signal ends up being shared out between many different channels, and may not be yet detected in any single one $[3,4]$. With $100 \mathrm{fb}^{-1}$ of integrated luminosity at $13 \mathrm{TeV}$ though, the 0 -lepton + jets $+p_{T}^{\text {miss }}$ searches should be sensitive to up to $1900 \mathrm{GeV}$.

We further combine the search strategies in the prompt and displaced channels to demonstrate that a much better sensitivity could be obtained by optimising cuts. In particular, we find that combining the relaxed DV cuts with the hard cuts from the 0 -lepton + jets $+p_{T}^{\text {miss }}$ analysis, a $>3 \sigma$ discovery can be made with $300-1000 \mathrm{fb}^{-1}$ data for a $2 \mathrm{TeV}$ gluino mass depending on the systematic background. We indicate how this situation could be improved significantly by also relaxing some of the prompt cuts. It is clear that an optimised analysis in a DV + jets $+p_{T}^{\text {miss }}$ channel will yield better sensitivity than for either search method alone and we strongly urge the experimental collaborations to pursue this further.

Acknowledgments The authors acknowledge the support of France Grilles for providing cloud computing resources on the French National Grid Infrastructure. This work has been partially supported by STFC Grant ST/L000385/1, by the Office of High Energy Physics of the U.S. Department of Energy under Contract DE-AC02-05CH11231, by the National Science Foundation under Grant PHY-1316783, by the Foundation for Polish Science through its programme HOMING PLUS, by National Science Centre under research Grant DEC2014/15/B/ST2/02157, by the ILP LABEX under reference ANR-10LABX-63, and by French state funds managed by the ANR within the Investissements d'Avenir programme under reference ANR-11IDEX-0004-02. GC was funded by the postgraduate Conicyt-Chile Cambridge Scholarship 84130011. ND was partially supported by the Alexander von Humboldt Foundation. MB acknowledges support from the Polish Ministry of Science and Higher Education (Decision No. 1266/MOB/IV/2015/0). BCA, MB and GC would like to thank other members of the Cambridge SUSY Working group for discussions. RZ thanks B. Fuks, A. Mariotti, D. Redigolo and O. Slone for useful discussions. We thank the authors of Delphes 3 for discussions about the program related to this work. ND and MB would like to thank the Cavendish Laboratory for hospitality offered while working on this project. MB and RZ thanks the Galileo Galilei Institute for Theoretical Physics and INFN for hospitality and partial support during the completion of this work.

Open Access This article is distributed under the terms of the Creative Commons Attribution 4.0 International License (http://creativecomm ons.org/licenses/by/4.0/), which permits unrestricted use, distribution, and reproduction in any medium, provided you give appropriate credit to the original author(s) and the source, provide a link to the Creative Commons license, and indicate if changes were made.

Funded by SCOAP ${ }^{3}$.

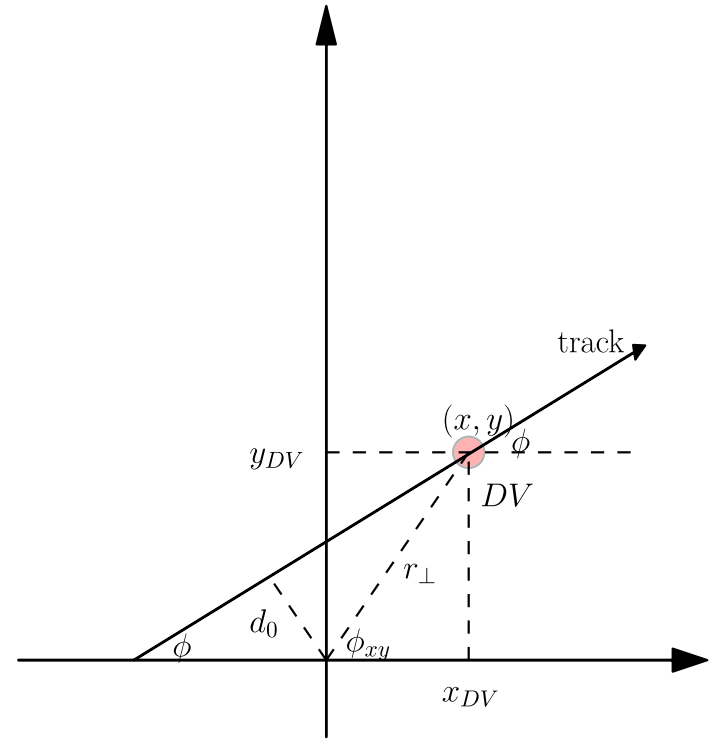

Fig. 12 Schematic view in the transverse $x-y$ plane of a displaced decay. The transverse impact parameter $d_{0}$ is defined with respect to the origin $(0,0,0)$. The daughter particle, which forms the track, was produced at $(x, y, z)$ from the decay of a long-lived particle. The DV position is reconstructed from the average of all track's production vertices, represented by the pink disc

\section{Appendix A: Definitions of displaced observables}

Here we define the relevant observables used in the DV recast. When a displaced track is produced at a point ${ }^{7}(x, y, z)$, we define the transverse distance of the truth track's production vertex to be

$r_{\perp}=\sqrt{x^{2}+y^{2}}$.

Each track will have a transverse impact parameter $d_{0}$, which corresponds to the distance of closest approach of the track to the origin $(0,0,0)$ in the $x-y$ plane:

$d_{0}=r_{\perp} \times \sin \left(\phi_{x y}-\phi\right)$,

where $\phi$ is the azimuthal angle of the track, such that $\tan \phi=p_{y} / p_{x}$ with $p_{x}, p_{y}$ the $x$ and $y$ component of the track momentum. $\phi_{x y}$ corresponds to the angle in the transverse plane of the trajectory of the mother displaced particle, as shown in Fig. 12.

Selected tracks are clustered together to form a DV. The DV position $\left(x_{\mathrm{DV}}, y_{\mathrm{DV}}, z_{\mathrm{DV}}\right)$ is defined to be the average position of all track's production points in that selected vertex. The DV position in the transverse plane is defined to be

$r_{\mathrm{DV}}=\sqrt{x_{\mathrm{DV}}^{2}+y_{\mathrm{DV}}^{2}}$

\footnotetext{
$\overline{7}$ The origin is defined to be the interaction point, and $z$ is along the beam line.
} 
The DV position ( $\left.x_{\mathrm{DV}}, y_{\mathrm{DV}}, z_{\mathrm{DV}}\right)$ is equal to the truth decay position of the mother particle in principle, but in our simulation it is defined from the displaced tracks, using the truth information for the production positions, as explained above.

\section{References}

1. G. Aad et al., JHEP 10, 054 (2015). doi:10.1007/JHEP10(2015)054

2. L.J. Hall, D. Pinner, J.T. Ruderman, JHEP 04, 131 (2012). doi:10. 1007/JHEP04(2012)131

3. G. Aad et al., JHEP 10, 134 (2015). doi:10.1007/JHEP10(2015)134

4. A. Barr, J. Liu, arXiv:1605.09502 (2016)

5. J.L. Evans, M. Ibe, T.T. Yanagida, Phys. Lett. B 705, 342 (2011). doi:10.1016/j.physletb.2011.10.031

6. T. Jelinski, J. Pawelczyk, K. Turzynski, Phys. Lett. B 711, 307 (2012). doi:10.1016/j.physletb.2012.04.011

7. J.L. Evans, M. Ibe, S. Shirai, T.T. Yanagida, Phys. Rev. D 85, 095004 (2012). doi:10.1103/PhysRevD.85.095004

8. Z. Kang, T. Li, T. Liu, C. Tong, J.M. Yang, Phys. Rev. D 86, 095020 (2012). doi:10.1103/PhysRevD.86.095020

9. N. Craig, S. Knapen, D. Shih, Y.Zhao, JHEP 03, 154 (2013). doi:10. 1007/JHEP03(2013)154

10. M. Abdullah, I. Galon, Y. Shadmi, Y. Shirman, JHEP 1306, 057 (2013). doi:10.1007/JHEP06(2013)057

11. H.D. Kim, D.Y. Mo, M.S. Seo, Eur. Phys. J. C 73(6), 2449 (2013). doi:10.1140/epjc/s10052-013-2449-z

12. P. Byakti, T.S. Ray, JHEP 1305, 055 (2013). doi:10.1007/ JHEP05(2013)055

13. N. Craig, S. Knapen, D. Shih, JHEP 08, 118 (2013). doi:10.1007/ JHEP08(2013)118

14. J.A. Evans, D. Shih, JHEP 1308, 093 (2013). doi:10.1007/ JHEP08(2013)093

15. L. Calibbi, P. Paradisi, R. Ziegler, JHEP 1306, 052 (2013). doi:10. 1007/JHEP06(2013)052

16. T. Jelinski, JHEP 1309, 107 (2013). doi:10.1007/ JHEP09(2013)107

17. I. Galon, G. Perez, Y. Shadmi, JHEP 1309, 117 (2013). doi:10. 1007/JHEP09(2013)117

18. W. Fischler, W. Tangarife, JHEP 1405, 151 (2014). doi:10.1007/ JHEP05(2014)151

19. S. Knapen, D. Shih, JHEP 1408, 136 (2014). doi:10.1007/ JHEP08(2014)136

20. R. Ding, T. Li, F. Staub, B. Zhu, JHEP 1403, 130 (2014). doi:10. 1007/JHEP03(2014)130

21. L. Calibbi, P. Paradisi, R. Ziegler, Eur. Phys. J. C 74(12), 3211 (2014). doi:10.1140/epjc/s10052-014-3211-x

22. A. Basirnia, D. Egana-Ugrinovic, S. Knapen, D. Shih, JHEP 06, 144 (2015). doi:10.1007/JHEP06(2015)144

23. T. Jelinski, PoS CORFU2014, 089 (2015)

24. T. Jelinski, J. Gluza, Phys. Lett. B 751, 541 (2015). doi:10.1016/j. physletb.2015.11.003

25. S. Knapen, D. Redigolo, D. Shih, JHEP 03, 046 (2016). doi:10. 1007/JHEP03(2016)046

26. B. Allanach, M. Badziak, C. Hugonie, R. Ziegler, Phys. Rev. D 92(1), 015006 (2015). doi:10.1103/PhysRevD.92.015006
27. A. Delgado, G.F. Giudice, P. Slavich, Phys. Lett. B 653, 424 (2007). doi:10.1016/j.physletb.2007.07.057

28. G.F. Giudice, R. Rattazzi, Nucl. Phys. B 511, 25 (1998). doi:10. 1016/S0550-3213(97)00647-0

29. M. Badziak, M. Olechowski, S. Pokorski, JHEP 06, 043 (2013). doi:10.1007/JHEP06(2013)043

30. S. Schael et al., Eur. Phys. J. C 47, 547 (2006). doi:10.1140/epjc/ s2006-02569-7

31. M. Badziak, I. Dalianis, Z. Lalak, JHEP 02, 173 (2016). doi:10. 1007/JHEP02(2016)173

32. U. Ellwanger, C. Hugonie, Comput. Phys. Commun. 175, 290 (2006). doi:10.1016/j.cpc.2006.04.004

33. U. Ellwanger, C. Hugonie, Comput. Phys. Commun. 177, 399 (2007). doi:10.1016/j.cpc.2007.05.001

34. A. Buckley, Eur. Phys. J. C 75(10), 467 (2015). doi:10.1140/epjc/ s10052-015-3638-8

35. T. Sjöstrand, S. Ask, J.R. Christiansen, R. Corke, N. Desai, P. Ilten, S. Mrenna, S. Prestel, C.O. Rasmussen, P.Z. Skands, Comput. Phys. Commun. 191, 159 (2015). doi:10.1016/j.cpc.2015.01.024

36. M. Cacciari, G.P. Salam, G. Soyez, Eur. Phys. J. C 72, 1896 (2012). doi:10.1140/epjc/s10052-012-1896-2

37. B.C. Allanach, Comput. Phys. Commun. 143, 305 (2002). doi:10. 1016/S0010-4655(01)00460-X

38. B.C. Allanach, P. Athron, L.C. Tunstall, A. Voigt, A.G. Williams, Comput. Phys. Commun. 185, 2322 (2014). doi:10.1016/j.cpc. 2014.04.015

39. M. Muhlleitner, A. Djouadi, Y. Mambrini, Comput. Phys. Commun. 168, 46 (2005). doi:10.1016/j.cpc.2005.01.012

40. P.Z. Skands et al., JHEP 07, 036 (2004). doi:10.1088/1126-6708/ 2004/07/036

41. B.C. Allanach et al., Comput. Phys. Commun. 180, 8 (2009). doi: $10.1016 / j . c p c .2008 .08 .004$

42. ATLAS Collaboration, G. Aad et al., arXiv:0901.0512 (2009)

43. G. Aad et al., JHEP 09, 176 (2014). doi:10.1007/JHEP09(2014)176

44. ATLAS Collaboration, M. Aaboud et al., arXiv:1605.03814 (2016)

45. Z. Liu, B. Tweedie, JHEP 06, 042 (2015). doi:10.1007/ JHEP06(2015)042

46. C. Csaki, E. Kuflik, S. Lombardo, O. Slone, T. Volansky, JHEP 08 , 016 (2015). doi:10.1007/JHEP08(2015)016

47. Y. Cui, B. Shuve, JHEP 02, 049 (2015). doi:10.1007/ JHEP02(2015)049

48. C. Csaki, E. Kuflik, S. Lombardo, O. Slone, Phys. Rev. D 92(7), 073008 (2015). doi:10.1103/PhysRevD.92.073008

49. J.A. Evans, J. Shelton, JHEP 04, 056 (2016). doi:10.1007/ JHEP04(2016)056

50. A. Coccaro, D. Curtin, H. J. Lubatti, H. Russell, J. Shelton, arXiv: 1605.02742 (2016)

51. A. de la Puente, A. Szynkman, Eur. Phys. J. C 76(3), 124 (2016). doi:10.1140/epjc/s10052-016-3969-0

52. G. Aad et al., Phys. Rev. D 92(7), 072004 (2015). doi:10.1103/ PhysRevD.92.072004

53. V.M. Abazov et al., Phys. Rev. Lett. 103, 071801 (2009). doi:10. 1103/PhysRevLett.103.071801

54. G. Aad et al., JINST 8, P07015 (2013). doi:10.1088/1748-0221/8/ 07/P07015 